\title{
Gergely Guszmann
}

\section{La typologie des héros. Le saint, le héros, le grand homme et le martyr ${ }^{*}$}

\section{"Le Roi ne meurt jamais » : le roi et le saint}

Ernst H. Kantorowicz, le grand médiéviste allemand a esquissé, dans les années 1950, les attributs surnaturels des rois du Moyen Age. Chez Kantorowicz, les rois exercent un pouvoir sur la terre, semblable à celui de Dieu. Ce pouvoir descend de Dieu, est incontestablement divin et, par conséquent la personne du roi ne peut être jugée par personne. Toutes les hautes vertus sont désormais réservées au roi qui est le gouverneur du peuple et, comme il est à la Tête de l'Etat, son nom et sa personne politique sont immortels. Selon la conception de «deux corps de roi», le roi a à la fois un corps naturel, mortel, et un corps fictif créé par l’institution de la royauté. Au corps naturel survit le corps fictif qui, par son immortalité, justifie la formule «le Roi ne meurt jamais». ${ }^{2}$ Le corps politique du roi maintient donc la continuité institutionelle, et, contrairement à la personne naturelle, l'office du roi est éternel. Le caractère personnel du roi est aussi gardé par la mémoire (par les historiens, les poètes, les peintres, les sculpteurs etc.) et pendant l'époque de la Renaissance, l’allégorie des fêtes et des images associe souvent les actes héroïques gréco-romains aux triomphes contemporains. ${ }^{3}$

Au début du Moyen Age, l’illustration de l'empereur romain, qui était l'objet d’un culte, disparaît totalement. L'installation du christianisme (durant les $\mathrm{IV}^{\mathrm{e}}$ et le VII ${ }^{\mathrm{e}}$ siècles) entraîne un changement profond des dieux et des héros. L’Antiquité gréco-romaine honorait des personnages héroïques comme les héros, les dieux et les demi-dieux mais ces catégories se sont modifiées par l’apparition de la religion monothéiste remplaçant le polythéisme. A la suite de cette mutation, de nouvelles catégories se sont formées:

- le saints dont le culte, à un niveau supérieur, est acquis par leurs miracles; pour la

\section{$1^{*}$ https://doi.org/10.24361/Performa.2020.12.11}

La recherche a été soutenue par le projet « Développement complexe des capacités et des services de recherche à l’Université Károly Eszterházy EFOP-3.6.1-16-2016-00001».

2 Ernst Kantorowicz: Les deux corps de roi. Essai sur la théologie politique du Moyen Age, Paris, Gallimard, 1989.

3 Yael Zerubavel: Le héros national: un monument collectif. Tradition et politiques de commemoration. Exemples israéliens. In: Pierre Centlivres - Daniel Fabre - Françoise Zonabend (éds.): La fabrique des héros, Paris, MSH, 1998. 168-178. 
papauté le saint est devenu un héros incontesté ;

- le roi qui est un nouveau type de gouverneur du peuple. ${ }^{4}$

Dans le champ d'incarnation de l'héroïsme, les rois du Moyen Age conservent les trois fonctions des empereurs de l’Antiquité, ainsi les rois sont-ils à la fois juges, guerriers et bienfaiteurs. Les nouveaux héros plus révolutionnaires sont les saints médiévaux qui sont les héritiers des dieux et des génies. Tout au début, le saint est un témoin de sa foi, un martyr jusqu'au IV siècle, lorsque Théodose installe le christianisme comme religion de l'Empire Romain. Toutefois, le saint devient aussi un confesseur. Le type de saint en tant que confesseur devient fondamental dans l’idéologie chrétienne. ${ }^{5}$

Ce type médiéval de la sainteté s’est essentiellement modifié du $\mathrm{IV}^{\mathrm{e}}$ au $\mathrm{XV}^{\mathrm{e}}$ siécle. Les nouveaux saints sont alors uniquement les rois ou les princes qui convertissent leurs peuples. La sainteté n’est plus définie par des miracles mais par des vertus. Pour distinguer le vocabulaire de l’attribution de la sainteté à cette époque, Jacques LeGoff propose quatre notions essentielles dans le domaine du sacré:

- $\quad$ Premier terme: le saint qui marque une caractéristique substantiellement religieuse;

- Deuxième terme: le sacré, sembablement au saint, est proche du surnaturel mais est un attribut exclusivement réservé au roi, héritier d’un certain nombre de pratiques du pouvoir octroyé par Dieu;

- Troisième terme: le sacerdotal qui exprime l’héritage biblique, à côté des héritages indo-européens ou barbares;

- Quatrième terme: le thaumaturgique qui se rapporte au césaro-papisme de l'empire byzantin où le roi est à la tête de l’Eglise.

Considérons l’idée que se fait LeGoff du caractère sacré de l’attribution du roi, qu’il s'agisse des origines bibliques ou indo-européennes, et qui après avoir reçu l’onction du sacre par la cérémonie du couronnement, devenait un « envoyé » du Christ. Dès les XIe-XIIe siècles, c’est le roi, gouverneur du peuple, qui est devenu l'image de Dieu dans les textes de l'époque. Le roi est le représentant de Dieu sur la terre, couronné par Dieu (Deo coronatus). Il doit donc faire régner les deux principes sociaux fondamentaux du christianisme: la paix et la justice. Les insignes de son pouvoir, notamment le sceptre, le trône, l’anneau et la main de justice peuvent aussi représenter son caractère sacré. Malgré cela, ses premières fonctions à l'intérieur comme gouverneur du peuple, guerrier, bienfaiteur et juge ne sont pas des caractéristiques religieuses. En tant que tel, chez LeGoff, le caractère par excellence du roi médiéval ne peut être autre qu’un proto-type du héros laïc.

On peut dire à la limite que le roi est un maillon dans l'émergence lente de la laïcité en Occident.

4 Jacques LeGoff: Héros du Moyen Age, le Saint et le Roi, Paris, Gallimard, 2004.

5 Jacques LeGoff: L'Europe est-elle née au Moyen Age? Seuil, Faire l’Europe, 2003. 94. 
Quant aux saints, on sait qu'on a reconnu au XIX et au XX $X^{\mathrm{e}}$ siècle des saints laïcs. Par conséquent, ce recueil d'études qui vont à la recherche de la sainteté et de la sacralité dans les héros de l’Occident, est, d'une façon paradoxale, une investigation dans les racines lointaines de l'esprit laïc en Occident. ${ }^{6}$

En fait, c'est justement cette interprétation suggestive du caractère héroïque du roi qui me semble une étape très importante dans l'évolution de la signification historique du mot héros. Par ailleurs, le caractère du saint est aussi essentiel si nous considérons sa mémoire vivante dans la société. Les rois et les saints marquent leur pouvoir sur l'espace et sur le temps, donc leur mémoire est gardée pour l'avenir. Le saint marque doublement l'espace de son empreinte: premièrement, par un endroit qui l'emporte, c'est le lieu de sa tombe, deuxièmement, par ses reliques, créées après sa mort et cultivées par les fidèles. De même, les rois sont des marqueurs d'espace par leurs lieux de puissance et de royauté comme les châteaux, la capitale et la cour où se concentre la pratique du pouvoir.

Quant à leur empreinte dans le temps, l'on sait que l'on célèbre leur fête dans le calendrier. La personne, l’acte politique et le règne du roi sont enregistrés et marquent ainsi les années. La présence du saint dans le calendrier est notée comme les anniversaires des saints, autrement dit, le jour de la mort du saint devient un événement significatif. La mort des rois et des saints est encore plus remarquable que la mort des héros de l’Antiquité qui sont devenus des dieux ou des demi-dieux, et comme tels, quasi immortels.

En ce qui concerne les martyrs et les héros médiévaux, Jacques LeGoff montre merveilleusement que leur origine remonte au moment où il leur fut attribué un rôle exceptionnel. La dévotion de la sainteté s’est manifestée par le culte des reliques qui peuvent guérir celui qui les touche. Par conséquent, la présence sociale de la sainteté est fortement marquée comme une évocation de la résurrection dans la mémoire vivante. Il semble donc que ni le roi ni le saint ne meurent jamais.

\section{L’historicité du mot héros}

Aux siècles des Lumières, on peut prendre en compte, dans le champ notionnel du terme héros, une redéfinition de l'évolution ou, bien entendu, le remaniement terminologique du mot.

Suivant la classification terminologique proposée par Daniel Fabre, éditeur de l'un des ouvrages de synthèse les plus récents sur les théories de la fabrication des héros, on peut clairement distinguer trois remaniements conceptuels dans l'historiographie traitant de la question de l’héroïsation. Premier remaniement: dans le discours littéraire, le mot héros apparaît comme le personnage illustre du récit épique. Considérons dans les langues germaniques les Helden, protagonistes du récit qui sont distingués d'avec les Heroen, créatures de la mythologie. Le mot «héros» devient alors synonyme de « person6 LeGoff: Héros du Moyen Age, le Saint et le Roi. 1077. 
nage » d’un récit. En fait, à partir du XVIIIe siècle, ce héros de récit (ou de roman) est présenté comme «discriminateur ultime des valeurs». Deuxième remaniement: dans les décrets de Benoît XIV, la vertu exceptionnelle d'un saint consiste dans l'observation rigoureuse des règles catholiques. Troisième remaniement: il semble que depuis l'époque de la Renaissance, la portée du mot «héros», c'est-à-dire, un personnage militaire réel ayant obtenu un triomphe sanglant, ait été pragmatiquement reconsidérée. Dans ce discours, le contenu d'un acte violent devient plutôt ironique que héroïque, dans le sens d'un épithète pour dénoncer un attribut méritoire. Chez Voltaire, le personnage utile ou agréable est substantiellement plus précieux que les héros, et le nom de ce nouvel acteur est: «le grand homme».7

L'invention du grand homme $e^{8}$ apporte une modification dans le sens attribué jusque-là au mot «héros» mais l'exemplarité reste encore la vertu essentielle à la fois du «héros» et du «grand homme». Par ailleurs, l’image des vertus héroïques classiques et humanistes est fondamentalement changée pour évoquer la reconnaissance d'une utilité sociale. On pourrait conclure de ce point de vue voltarien que les œuvres du grand homme sont utiles pour la société et que, en revanche, le triomphe militaire du héros ne comporte aucune signification sociale. Cependant le grand homme, contrairement aux rois ou aux guerriers, est souvent ignoré avant d'être reconnu. Et puis, «les recueils de 'vie d'hommes illustres', dont Plutarque est toujours le parangon, le rite de l'éloge du grand homme que les académies adoptent (surtout après 1750), la multiplication des médailles, des images, des statuettes qui prennent place dans les intérieurs domestiques, la peinture d’histoire, les premiers monuments publics dédiés à des civils, les pèlerinages sur les lieux où vécurent, et parfois vivent encore, les personnes qu’honore la communauté européenne des esprits éclairés sont autant de signes où l’on a récemment reconnu un puissant mouvement de 'canonisation laïque'.»9

Ce nouvel héroïsme commenté ici devient une notion capitale du grand homme. On peut supposer que la définition d'utilité sociale du grand homme comme bienfaiteur de l'humanité soit homogène, mais il y a plusieurs autres dimensions dans la pratique. Notamment, le grand homme est un personnage patient qui peut précisément envisager un plan pour créer quelque chose de socialement utile. Il est donc l’antipode du héros passionné de l’Antiquité. Voilà le génie souvent admiré par certains penseurs des Lumières.

\section{La construction d'un panthéon laïc: " Aux grands hommes la patrie reconnaissante.»}

7 Pierre Centlivres - Daniel Fabre - Françoise Zonabend (éds.): La fabrique des héros. Paris, MSH, 1998.

8 Prendre l'expression de Fabre à Pierre Centlivres - Daniel Fabre - Françoise Zonabend (éds.): La fabrique des héros. 239.

9 Daniel Fabre: L’atelier des héros. In: Centlivres -Fabre-Zonabend (éds.): La fabrique des héros. 241. 
En suivant Fabre, on pourrait interpréter le changement terminologique et pragmatique de l’héroïsation comme un triangle dont chaque sommet désigne une autre étape dans la représentation pratique de l'homme et de sa grandeur entre la Révolution et la Restauration. Chez Fabre, le premier sommet du triangle représente l’influence révolutionnaire de la pensée des Lumières sur les grands hommes. Le second sommet représente la gloire temporelle incorporée par le triomphe militaire d'un personnage charismatique (ici Napoléon). Et le troisième sommet montre comment les penseurs politiques libéraux imaginent un type de héros comme une figure qui a des tensions inhérentes à la pratique du pouvoir.

Par conséquent, on met en forme un nouveau rituel. C'est alors que l'on fixe les grands noms pour la mémoire à travers l'idée de consacrer à Paris un temple (le Panthéon) aux grands hommes. Le Panthéon est construit afin de garder l'esprit des grandes âmes pour l'avenir. En fait, à examiner les grands hommes panthéonisés, les catégories écrites par Marat dans L’Ami du peuple et citées par Fabre, me semblent merveilleusement représentatives:

le guerrier qui expose sa vie, le philosophe qui éclaire la nation, le législateur qui lui donne de bonnes lois, le magistrat qui les fait exécuter avec intégrité, l’orateur qui épouse avec zèle la défense des opprimés, le négociant généreux qui ramène l’abondance dans les temps de disette. ${ }^{10}$

Pourtant, à côté des grands acteurs de la Nation, les petits personnages, c'est-à-dire les soldats, de l'histoire française jouent un rôle remarquable dans le processus de la panthéonisation. Selon Fabre, en considérant les figures emblématiques du Panthéon, il y a deux modes pour définir les héros: les philosophes qui sont les pères fondateurs de la Nation, les acteurs militaires et les victimes converties en martyrs.

\section{Le martyr et le héros: la souffrance et le sacrifice du héros}

L'un des nombreux aspects soulignés dans les approches classiques du concept du héros est sa nature «charnelle et éternelle», au sens où les notions jouent un rôle important dans la légitimation du pouvoir par les groupes dominants. L’une des formes les plus efficaces de la domination idéologique se produit lorsque les groupes dominés construisent les héros dominants comme naturels.

Afin d’abréger ce survol historique, j'utiliserai dans ce sous-chapitre une conception particulière de la notion du héros. Ma définition du héros est la suivante: un héros est une figure illustre porteur de valeurs glorifiées par un groupe et qui déploie une action historique réussie pour le bénéfice de la société. Ces idées de groupes peuvent être évaluées positivement, négativement ou ne pas être évaluées du tout, et ce en fonction du point de vue de l'appartenance à un groupe ou de l'éthique de chacun. Cela étant, je

10 Daniel Fabre: L’atelier des héros. 245. 
n’identifie pas exclusivement les valeurs et les actions concrètes des héros. Le choix de la construction d’un héros est plus généralement associé aux groupes sociaux, aux classes, aux castes ou aux communautés. Il représente leurs intérêts fondamentaux. La théorie rendant compte des différentes croyances de la naissance et du caractére du héros est complexe, et peut être résumée comme suit (pour des précisions et d’autres références, voir Centlivres - Fabre - Zonabend, 1998.)

- Par exemple, les noms des rues portent le nom d'un homme exemplaire permettant ainsi de garder celui-ci dans la mémoire collective;

- Un héros est l'acteur d'un geste sacrificiel pour la patrie en danger;

- Tout d'abord, le héros est un «personnages historique» avec un nom inscrit dans une multiplicité de textes et son individualité est toujours documentée;

- Les héros légendaires peuvent devenir un emblème unificateur pour une communauté, et les héros actuels peuvent incarner leur résurrection;

- Le héros peut apparaître comme le représentant du «caractère national» avec une personnalité mystique, l’archétype de l'ethnie. Mais, selon Krzysztof Pomian, après la chute des idéologues chauvinistes du XXe siécle, le patrimoine culturel, les lieux et mémoriaux des héros se substituent à l'esprit national en tant que facteurs unificateurs. de «l'esprit» national. ${ }^{11}$ Ensuite le patrimoine culturel, les lieux et mémoriaux des héros remplacent la fonction du facteur unificateur;

- Le poète est un reflet de la gloire patriotique ayant une position centrale en littérature et luttant pour une cause populaire dans la sphère publique. Par exemple, le poète héroique est celui qui lutte contre le despotisme d'un pouvoir occupant ;

- Le caractére des «héros des sciences». Par exemple, ce terme désigne le savant qui fait une découverte scientifique au service de l’humanité;

- Le vainqueur de la guerre patriotique est l'exemple le plus approprié;

- Le caractère de l’antihéros n’est pas l’antipode du héros mais, selon Pierre-Yves Bourdil ${ }^{12}$, la figure du fou, l’homme sans qualité et sans vertus héroiques. Par exemple, le figure satirique du soldat guerrier de Jaroslav Hašek dans Le brave soldat Chvéik.

Telles sont quelques-unes des principales propriétés des lectures du héros et du martyr selon la théorie multidisciplinaire. Ainsi, les perspectives constituent la base «axiomatique» des représentations sociales d'un groupe et contrôlent - à travers des attitudes sociales spécifiques et des modèles mentaux personnels - les discours des individus et les autres pratiques sociales des membres du groupe. De cette manière, les notions de héros constituent la ressource nécessaire à la coopération, la coordination et la 11 Krzysztof Pomian: Nation et patrimoine. In Daniel Fabre (éd.): L'Europe entre cultures et nations. Paris, Éd. de la MSH, 1996. 85-95.

12 Pierre-Yves Bourdil: Les Miroirs du moi: les héros et les fous, Paris, Édition du CNRS, 1987. 
cohésion au sein du groupe, ainsi qu’à la gestion des relations de compétition, de conflit ou de lutte entre les groupes. Ce n'est que dans une telle théorie que nous pouvons rendre compte du discours idéologique et d’autres pratiques sociales, à savoir des éléments dérivés de représentations sociales basées sur l’idéologie et de l’instanciation des relations sociales entre groupes.

\section{Le champ de héros et de martyr}

Dans ce chapitre, je fais un tour de l'horizon des théories du traitement des notions de héros et de martyr. Je me penche ensuite sur la distinction entre l'usage des termes: héros et martyr. Pour commencer, je tiens à dresser un tableau des caractéristiques de ces mots. Nous pouvons placer ces notions dans le champ des interprétations scientifiques et des usages politiques. En effet, de nombreuses questions sont soulevées dans cette approche. Par exemple, si dans un contexte politique, il est facile de trouver ces mots, comment peut-on adapter l'interprétation de ces mots au pouvoir? Est-ce que les mêmes notions revêtent un sens différent selon les groupes sociaux et politiques? Quels sont les attributs des notions qui sont transmis par un groupe? Ces questions sont illustrées par les différentes constructions des mots héros et martyr.

\section{LE HÉROS}

- le courage militaire

- le vainqueur/le guerrier

- représentant exemplaire

- $\quad$ successeur des vertus du roi/chevalier

- $\quad$ sacrifice volontaire

- $\quad$ les grandes ancêtres

\section{CHAMP COMMUN DE}

LA GRANDEUR

- la nation comme espace de reconnaissance et comme lieu d'application de l'activité du héros/martyr

- fondateur de dynasties ou d'États

- la gloire nationale imposée par le «haut»/la gloire locale constituée par le «bas»

- l'agent prédestiné à un «destin national»

- souffrance personnelle au service d'un objectif jugé insignifiant

\section{LE MARTYR}

- $\quad$ risque de la mort tragique

- la manifestation la plus probante d'une vertu héroïque

- successeur des vertus du saint avec une loi divine

- sacrifice pour la communauté espace de solidarité entre les vivantes . 
Ce tableau n'est qu'une interprétation tenant compte à la fois du caractère relativement stable des notions et de leurs aspects flexibles, dynamiques, changeants, contextualisés et subjectifs. La première dimension s’explique en termes de représentations morales cultivées par des groupes, socialement partagées et relativement stables. En ce qui concerne la deuxième dimension, il s’agit de modèles mentaux spécifiques et subjectifs des membres du groupe ou de la société, qui contrôlent le discours et d’autres pratiques sociales dans une situation donnée. Dans les première et troisième rubriques il y a des paramètres généraux du héros et du martyr typique. Quant à la deuxième rubrique, il y a un espace où le héros et le martyr peuvent être situés par rapport à l’importance de leur action historique ou en mesure de leurs qualités extraordinaires. ${ }^{13}$ Selon Reinhart Koselleck, les vivants ont commencé à accepter la mort de leurs camarades en donnant une raison à leur mort et ce fut justement la fonction des monuments après la Première Guerre mondiale. ${ }^{14}$ Les morts étaient interprétées comme des actions exemplaires et les monuments aux morts ont revêtu un caractère métaphysique. L’installation de monuments n’est pas un événement neutre mais, d'une certaine facon, participe à la création d'une identité politique et d'une légitimité. C’est pour cette raison que les monuments fonctionnent comme des symboles dans l'espace public. La création ou l'érection de ces monuments n’est pas qu’un déplacement physique mais tout un rite public qui sert à la représentation de l’heroïsme moderne.

\section{Références}

Jean-Pierre ALBERT: Du martyr à la star. Les métamorphoses des héros nationaux. In: CENTLIVRES - FABRES - ZONABEND (éds.): La fabrique des héros, Paris, MSH, 1998. 12-31.

Pierre-Yves BOURDIL: Les Miroirs du moi: les héros et les fous, Paris, Édition du CNRS, 1987.

Pierre CENTLIVRES - Daniel FABRE - Françoise ZONABEND (éds.): La fabrique des héros. Paris, MSH, 1998. https://doi.org/10.4000/books.editionsmsh.3993

Daniel FABRE: L’atelier des héros. In: CENTLIVRES - FABRE - ZONABEND (éds.): La fabrique des héros. Paris, MSH, 1998.

Daniel FABRE (éd.): L'Europe entre cultures et nations. Paris, Éd. de la MSH, 1996. https://doi.org/10.4000/ books.editionsmsh.3898

Reinhard JOHLER: « Malheur au peuple qui a besoin de héros » ou pourquoi les Autrichiens n’ont-ils 13 Jean-Pierre Albert: Du martyr à la star. Les métamorphoses des héros nationaux. In: Centlivres -Fabre -Zonabend (éds.): La fabrique des héros. 12-31. Reinhard Johler: « Malheur au peuple qui a besoin de héros » ou pourquoi les Autrichiens n’ont-ils pas besoin de héros nationaux? In: Centlivres -Fabre -Zonabend (éds.): La fabrique des héros. 212-295. 14 Reinhart Koselleck: Kriegerdenkmale als Identitätsstiftungen der Überlebenden. In Odo Marquard- Karlheinz Stierle (éds.): Identität. München, Fink, 1979. 255-276. (voir) Antoine Prost: Les monuments aux morts. Culte républicain? Culte civique? Culte patriotique? In: Pierre Nora (éd.): Les lieux de mémoire. La République. Paris, Gallimard, Coll. Quarto I., 1997. 199-223. 
pas besoin de héros nationaux? In: CENTLIVRES -FABRE -ZONABEND (éds.): La fabrique des héros. 212-295.

Ernst KANTOROWICZ: Les deux corps de roi. Essai sur la théologie politique du Moyen Age, Paris, Gallimard, 1989.

Reinhart KOSELLECK: Kriegerdenkmale als Identitätsstiftungen der Überlebenden. In: Odo MARQUARD - Karlheinz STIERLE (éds.): Identität. München, Fink, 1979. 255-276.

Jacques LEGOFF: Héros du Moyen Age, le Saint et le Roi, Paris, Gallimard, 2004.

Jacques LEGOFF: L’Europe est-elle née au Moyen Age? Seuil, Faire l’Europe, 2003.

Odo MARQUARD - Karlheinz STIERLE (éds.): Identität. München, Fink, 1979.

Pierre NORA (éd.): Les lieux de mémoire. La République. Paris, Gallimard, Coll. Quarto I., 1997.

Krzysztof POMIAN: Nation et patrimoine. In: Daniel FABRE (éd.): L’Europe entre cultures et nations.

Paris, Éd. de la MSH, 1996. 85-95. https://doi.org/10.4000/books.editionsmsh.3917

Antoine PROST: Les monuments aux morts. Culte républicain? Culte civique? Culte patriotique? In: Pierre NORA (éd.): Les lieux de mémoire. La République. Paris, Gallimard, Coll. Quarto I., 1997. 199-223. Yael ZERUBAVEL: Le héros national: un monument collectif. Tradition et politiques de commemoration. Exemples israéliens. In: CENTLIVRES - FABRES - ZONABEND (éds.): La fabrique des héros, Paris, MSH, 1998. 168-178. 